\title{
Monocular and dichoptic interaction between afterimages
}

\author{
N. J. WADE \\ University of Dundee, Dundee DD1 $4 H N$, Scotland
}

\begin{abstract}
Two experiments were conducted in which an afterimage of a foveal grating in the right eye was observed either on its own or surrounded by a second afterimage. The separation and orientation of the lines comprising the surrounding afterimage, and the eye to which it was presented, were varied. The total duration of visibility of the central afterimage was reduced significantly when the dichoptic surround was separated from it by less than $.5 \mathrm{deg}$. This interocular suppression was independent of the orientation of the lines in the surround afterimage. Presentation of a monocular surround increased the duration of visibility of the central afterimage only when the orientations of the lines in the center and surround were the same. Thus, two processes were operating to produce this pattern of results: interocular suppression and intraocular contour facilitation that occurs for lines in the same orientation.
\end{abstract}

The visual systems of higher mammals are organized to integrate centrally the information from the two monocular projections (see Bishop \& Henry, 1971). Similar processes of binocular integration have been demonstrated in humans, both in terms of stereopsis (Julesz, 1971; Ogle, 1964) and in terms of binocular summation (Blake $\&$ Fox, 1973). A recent addition to the methods used for investigating binocular interaction involves the observation of prolonged, patterned afterimages (Atkinson, 1972, 1973; Forde, 1971; Wade, 1972, 1974). In general, afterimages comprised of line elements undergo sequences of fragmentation, disappearances, and reappearances. It is possible to examine the patterning of such sequences when afterimages are presented monocularly and dichoptically. This enables the determination of the extent to which the contours interact in the absence of any contour displacements as a consequence of eye movements. For example, the pattern of visibility of paired afterimages differs if they are presented monocularly or dichoptically (Atkinson, 1972): they remain visible simultaneously for longer when they are viewed monocularly. Moreover, the duration of simultaneous visibility was dependent upon the relative orientations of the two lines under both viewing conditions. The results suggested that two processes were operating; one involved suppression between the eyes and the other involved facilitation within an eye. This last process was orientation-specific in that it occurred only for paired lines in the same orientation.

The present experiments examined the same problem of binocular interaction between afterimages. Rather than using single lines, the afterimages were of gratings. In both experiments, a

This research was supported by a grant from the Science Research Council. The assistance of Kirsteen Greig in the collection and analysis of the data is gratefully acknowledged. 1-deg grating was presented foveally to the right eye, and this was surrounded by an annular afterimage. The annular surround was presented to the left eye in Experiment $I$ and both monocularly and dichoptically in Experiment II. Factors such as the separation between the central afterimage and the surround, and the orientation of the lines comprising the surround, were varied in the experiments. The central grating was vertical and had a spatial frequency of 4 cycles/deg in both experiments.

\section{EXPERIMENT I}

This experiment was concerned with dichoptic interaction alone. The right eye was always presented with a small vertical grating and the status of the stimuli in the other eye was manipulated (see Figure 1). A reference condition involved the vertical grating presented monocularly (Condition F, Figure 1), with no afterimage in the other eye. The other conditions involved dichoptic annular surrounds comprised of horizontal lines (A-D) or an annulus (E). The areas of all the horizontal surrounds were equated so that the energy in the stimuli which induced the afterimages would be equal; the same energy was presented in the annulus $E$, as the area was twice that of surround $A$, but it was masked by a $50 \%$ neutral density filter. The internal diameters of the annuli varied from 1.25 to $2.0 \mathrm{deg}$ so that the spatial characteristics of any interaction could be determined.

\footnotetext{
Method

Apparatus. The apparatus was essentially similar to that used previously (Wade, 1974). It consisted of a modified prism stereoscope, with adjustable biprisms for stimulus alignment. The gratings were cut out from black masking card and were mounted directly in front of a Multiblitz Report flash gun, which delivered $120 \mathrm{~J}$ in $2 \mathrm{msec}$. The stimuli used are shown in Figure 1 . The vertical grating subtended $1 \mathrm{deg}$ at the eye with a spatial frequency of 4 cycles $/ \mathrm{deg}$. The inner diameters of the annuli A-D were 2.0 ,
} 


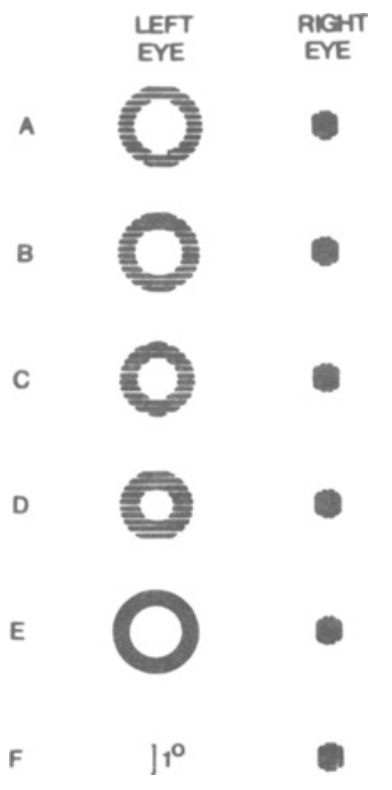

Figure 1. Stimulus configurations employed in Experiment I.

1.75. 1.50, and $1.25 \mathrm{deg}$, respectively, and for annulus $E$ it was $2.0 \mathrm{deg}$. The areas of the apertures were constant for A-D and half that of $E$, which was masked by a $50 \%$ neutral density filter. A microswitch was placed in front of the subject, and this was connected to a timer and an event recorder.

Subjects. Eight subjects participated in the experiment. All had normal or corrected vision.

Procedure. The task under all conditions involved depressing the switch when any part or all of the vertical afterimage was visible. Each subject was given a practice trial with one of the dichoptic configurations, and this was followed by 10 min dark adaptation. The stimuli were dimly illuminated prior to generation of the afterimages in order to enable appropriate binocular alignment, with the vertical grating in the center of the annular surround. Following discharge of the flash gun, the afterimages were observed for $2 \mathrm{~min}$ with the eyes closed in the darkened room. The intertrial interval was 5 min, during which time the subject was further dark adapted. The configurations were presented in random order within a session and each subject was tested in four sessions.

\section{Results and Discussion}

The results are summarized in Table 1 , in terms of the total duration of visibility of the vertical grating, its frequency of disappearance, the periods of visibility (duration/frequency), ${ }^{1}$ and the time from the initial visibility of the afterimage to its first and also to its last disappearance.
The grating afterimage remained visible for longer when it was presented on its own. The overall $F$ ratio from an analysis of variance indicated that the means differed significantly (see Table 1). Post hoc comparisons between the means were made using the method described by Rodger (1965). Means differing by more than $8.4 \mathrm{sec}$ do so significantly at the .05 level. Thus, the duration of visibility of the afterimage when viewed alone differs significantly from those dichoptic conditions involving a separation of less than $0.5 \mathrm{deg}$ (B-D). Conditions $\mathrm{A}$ and $\mathrm{E}$, having separations of $0.5 \mathrm{deg}$ between the inner contours of the surround and the outer contours of the center, did not differ significantly from $F$, although they were shorter in duration. The choice of this separation for the noncontoured control was unfortunate as it failed to demonstrate whether dichoptic suppression differs for contoured or noncontoured surrounds of equal energy. It did, however, show that the suppression effect does not operate at separations of $0.5 \mathrm{deg}$.

The frequencies of disappearance of the central afterimage differed significantly over the six conditions. The central afterimage disappeared significantly less frequently when viewed on its own (condition $F$ ) than when surrounded dichoptically. There were no significant differences between the frequencies under the dichoptic conditions. The periods of visibility (i.e., the average phases of visibility determined by duration/frequency) evidenced the differences expected from the above measure. The periods were significantly longer in the monocular condition ( $F$ ) than under any of the dichoptic conditions.

One of the most clearly differentiating features of the data concerned the duration of initial visibility of the afterimage, i.e., the time from its initial visibility to its first disappearance. For the single afterimage, this was about $40 \mathrm{sec}$, whereas for all the dichoptic conditions, the values were around $10 \mathrm{sec}$. This effect was highly significant.

The final measure, the time from the initial visibility to the final disappearance, did not differ significantly over the six conditions. This result indicates clearly that the suppression of the afterimage in the dichoptic conditions B-D was not occurring when the apparent intensity of the afterimage was at its lowest, but throughout its "life."

Table 1

Response Measures for the Visibility of Afterimages in Experiment I

\begin{tabular}{|c|c|c|c|c|c|c|c|}
\hline & $\mathbf{A}$ & B & $\begin{array}{c}\text { Stimulus } \\
\mathrm{C}\end{array}$ & $\begin{array}{c}\text { Configuration } \\
\text { D }\end{array}$ & $\mathbf{E}$ & $\mathrm{F}$ & F Ratio \\
\hline Duration (sec) & 61.1 & 56.9 & 56.7 & 54.6 & 65.4 & 68.2 & $6.15^{*}$ \\
\hline Frequency & 8.6 & 7.4 & 8.6 & 9.0 & 8.3 & 4.5 & $11.87 *$ \\
\hline Period (sec) & 9.1 & 9.9 & 8.0 & 7.2 & 10.2 & 30.0 & $4.84^{*}$ \\
\hline Time to first disappearance $(\mathrm{sec})$ & 7.8 & 10.7 & 7.3 & 4.6 & 10.7 & 38.2 & $8.59 *$ \\
\hline Time from first visibility to last disappearance (sec) & 87.8 & 84.4 & 84.7 & 86.5 & 89.0 & 82.8 & 1.48 \\
\hline
\end{tabular}

${ }^{*} p<.001$ 
Thus, the visibility of an afterimage in one eye can be suppressed significantly by the presence of a surrounding afterimage in the other eye, providing that the separation between the contours is less than $0.5 \mathrm{deg}$.

\section{EXPERIMENT II}

The interocular suppression demonstrated in the previous experiment was produced by orthogonal contours. This experiment examined the effect of contour orientation upon interocular suppression.

A vertical grating in the right eye was surrounded by a horizontal, 45-deg, or vertical annulus in the left eye, at a contour separation of $0.25 \mathrm{deg}$. In addition, the same configurations were presented monocularly in order to compare monocular with dichoptic interaction effects. The central grating was presented on its own as a reference condition (see Figure 2). Measures of the visibilities of the central and surround afterimages were obtained in order to derive more detailed information about the contour interactions.

\section{Method}

Apparatus. The apparatus was essentially similar to that used in the previous experiment. The stimulus configurations are shown in Figure 2. The central vertical grating subtended $1 \mathrm{deg}$ at the eye, with a spatial frequency of 4 cycles/deg. The inner diameter of the surrounds was $1.5 \mathrm{deg}$, so that the contour separations were $0.25 \mathrm{deg}$. Two microswitches were placed in front of the subjects, and these were connected to timers and separate channels of an event recorder.

Subjects. Ten subjects participated in the experiment. All had normal or corrected vision.

Procedure. The procedure for generating and observing the afterimages was essentially similar to that described in Experiment I. A practice trial was given with one of the configurations. followed by $10 \mathrm{~min}$ dark adaptation. Under the dichoptic conditions, the subject initially aligned the dimly illuminated stimuli prior to discharge of the flash gun. In the monocular conditions, an eye patch was placed over the left eye and the right eye fixated the dimly illuminated configuration before the afterimages were generated. The afterimages were observed for 2 min with the eyes closed and in darkness. The intertrial interval was $5 \mathrm{~min}$. The subject's task was to depress one switch for the visibility of all or part of the central vertical afterimage and the other for visibility of all or part of the surround. Clearly, both switches could be depressed simultaneously. The single afterimage trial was presented either initially, in the middle, or at the end of the series, and the three monocular and dichoptic conditions were successive. Each subject was tested in two sessions.

\section{Results and Discussion}

The data for each subject were averaged over sessions, and the group means are given in Table 2 for the visibility of the central and surrounding afterimage and for their simultaneous visibility.

Considering first the duration measures, the interocular suppression effect found in the previous experiment has been confirmed. Indeed, the effect here is greater than that found in Experiment $I$ : the single afterimage (G) was visible for about $20 \mathrm{sec}$

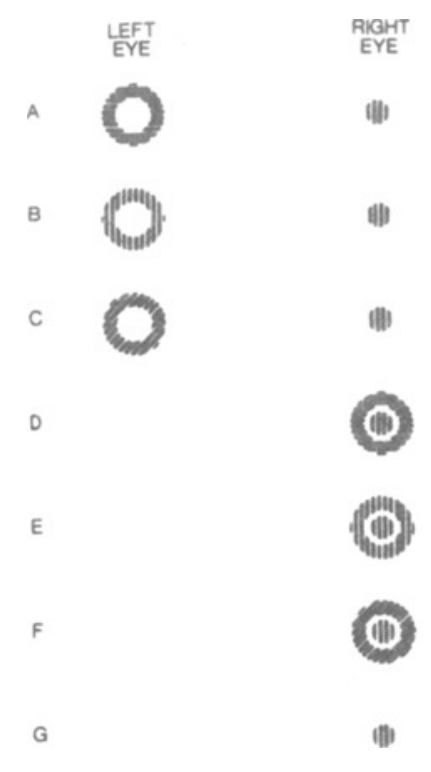

Figure 2. Stimulus configurations employed in Experiment II.

longer than the central dichoptic afterimages. Moreover, the interocular suppression was not influenced by the orientation of the surround. The durations of visibility of the central afterimages were longer in the monocular surround conditions (D-F) than for the single afterimage (G). Post hoc comparisons indicated that the single afterimage was visible for significantly shorter than that with the vertical monocular surround $(E)$, but did not differ significantly from the horizontal or 45-deg surround conditions ( $D$ and $F$ ). Thus, there appears to be an orientation-specific contour facilitation effect operating with the monocular surrounds and a nonorientation-specific suppression effect operating dichoptically.

The durations of visibility of the surround afterimages were significantly longer in the monocular than in the dichoptic conditions. These findings regarding the center and surround durations suggest that considerable binocular rivalry was operating in the dichoptic condition, with mutual suppression between the center and the surround. This suggestion is strongly supported by a comparison of the durations of simultaneous visibility under monocular and dichoptic viewing conditions (both measures in Table 2): both afterimages were visible together for about twice as long under the monocular conditions relative to the dichoptic.

The central afterimages disappeared less frequently under the monocular conditions than the dichoptic, but least frequently of all with the single afterimage. However, only the difference between the frequencies of the monocular and dichoptic conditions achieved statistical significance. The periods of visibility differed significantly over conditions, being longer for 
the monocular central afterimages than under dichoptic viewing. The period of the single afterimage did not differ significantly from that for the central afterimages under the monocular conditions. The surround periods and those for simultaneous visibility were also much longer for the monocular conditions.

The central afterimages had much longer latencies to the first disappearance with monocular viewing. and a similar, though less pronounced, effect was evident for the surrounds. However, the conditions were similar with regard to the time that elapsed before both central and surround afterimages disappeared simultaneously.

Finally, the times from the first visibility to the final disappearance of the afterimages differed. The single afterimage $(G)$ did not differ significantly from those central afterimages presented dichoptically, confirming the reult of Experiment I. The monocular afterimages remained visible for significantly longer than the dichoptic ones, both in terms of the central and in terms of the surround components. This result is indicative of some mutual facilitation under the monocular conditions.

The results of this experiment indicate that the visibility of an afterimage in one eye can be suppressed by a surrounding afterimage in the other eye regardless of its orientation, or enhanced by a similarly oriented afterimage in the same eye.

\section{GENERAL DISCUSSION}

The results of the above experiments are similar, in several respects, to those reported by Atkinson (1972) with afterimages of single lines or pairs of lines. She found that the duration of visibility of a line afterimage in one eye could be reduced by the presence of an afterimage in the other eye, and that this interocular suppression effect was independent of the orientations of the single lines. Atkinson (1972) also found that the duration of visibility of a line afterimage could be increased by the presence of a similarly oriented one in the same eye. This effect, which she called "facilitation," operated up to line separations of $1 \mathrm{deg}$. An analogous effect was found in Experiment II-a vertical grating afterimage surrounded by another vertical grating was visible for significantly longer than an equivalent vertical grating on its own. Atkinson's (1972) third finding was not, however, corroborated in the present experiments. She found that the duration of visibility of a line afterimage in one eye could be significantly reduced by the presence of a differently oriented afterimage in the same eye. This effect was referred to as "inhibition." It was not observed in the present experiments: surrounding a vertical grating with a horizontal or 45-deg grating had no significant effect upon the duration of visibility of the central grating relative to an equivalent grating on its own (Experiment II, conditions D, F, and G). Thus, no "inhibition" was recorded; in fact, the durations of visibility in the differently oriented surround conditions (D and F) were suggestive of "facilitation" rather than of "inhibition," but not significantly so. The differences between these results and those of Atkinson (1972) might have been due to the different afterimage configurations employed (in her experiments, similarly oriented vertical lines were aligned and horizontal lines were parallel), or the retinal locations stimulated (in her case, the afterimages were 2 deg parafoveal, whereas in the present experiments, the central afterimages were foveal).

The pattern of fragmentations and disappearances of afterimages comprised of lines are systematic and often involve lines disappearing and reappearing in

Table 2

Response Measures for the Visibility of the Central and Surround Afterimages and Their Simultaneous Visibility in Experiment II

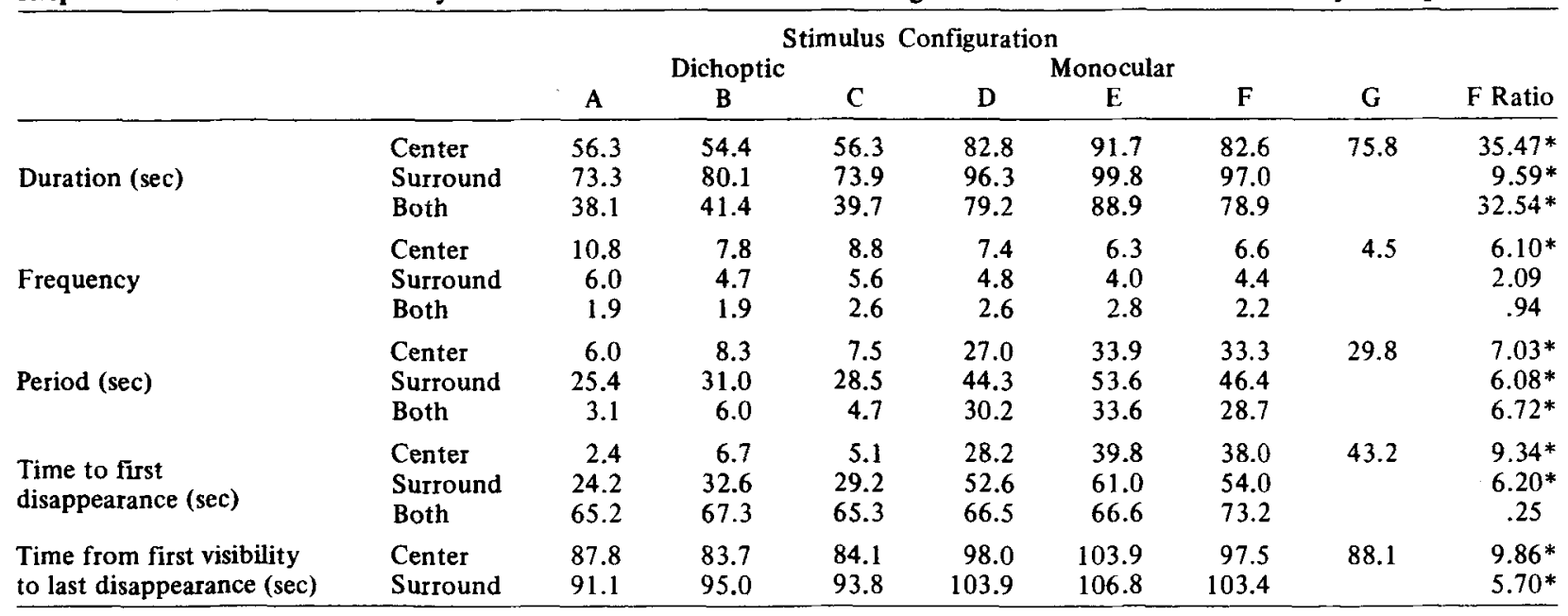

${ }^{*} p<.001$ 
unitary form. For example, an outline square will often fragment leaving 3,2, or 1 lines visible. This observation has led to the interpretation of results in terms of physiological, orientation-specific analyzers: these could undergo phases of adaptation and recovery, the phenomenal correlates of which would be the disappearances and reappearances of the line afterimages. The interactions between paired line afterimages can be similarly formulated in terms of facilitatory or inhibitory effects, which are dependent upon the relative orientations of the lines, and the eye(s) to which they are presented (Atkinson, 1972. 1973). If this type of interpretation is correct, it would be expected that the relative effects of a surround on the visibility of an afterimage should be similar to those on the response characteristics of orientationspecific analyzers measured physiologically under similar conditions. Such a comparison can be made, as Blakemore and Tobin (1972) recorded from a single cell in cat cortex while its receptive field was surrounded by annular gratings. They first determined the preferred orientation of a single cell and then measured its firing rate either on its own or surrounded by a grating that could be varied in orientation. The firing rate was reduced when the surround grating was in or around the same orientation as that of the single cell. Thus, the output of the cell was inhibited by the presence of a surround grating in and around its preferred orientation. The stimuli were all presented to one eye of the cat, and so that conditions could be likened to the monocular conditions (D-F) in Experiment II. However, it will be noted that the results of this experiment indicated that monocular afterimages were visible for longer when surrounded by similarly oriented gratings. The physiological index of such an increase in visibility would be facilitation. whereas the results indicated that the cat's cell was inhibited. Clearly, the psychophysical results do not find any interpretive support fron these physiological data, rather the reverse. Such a discrepancy dictates caution in equating the phenomenal characteristics of afterimage visibility with the operation of physiological analyzers.

The discussion of interocular suppression and intraocular facilitation has been confined mainly to the measures of afterimage duration. It is evident from Experiment $I$ that the various response indices are not perfectly correlated. For example, the duration measures for conditions $\mathrm{A}, \mathrm{E}$, and $\mathrm{F}$ (those with surround separations of $.5 \mathrm{deg}$ and the single afterimage) do not differ significantly. However, their frequencies of disappearance. periods of visibility and their times to the first disappearance all differ between the conditions (see Table 1). The measures for the dichoptic conditions at a separation of $.5 \mathrm{deg}$ are essentially similar to the other dichoptic conditions with smaller separations. Consequently, it is highly probable that dichoptic interaction is taking place for afterimages separated by $.5 \mathrm{deg}$, but this is not expressed significantly in the duration measure.

In summary, the duration of visibility of an afterimage can be reduced significantly by surrounding it with an afterimage in the other eye, provided that the separation between the afterimages is less than $.5 \mathrm{deg}$ visual angle. This interocular suppression is independent of the orientation of the surround relative to that of the central grating. Surrounding a foveal grating in the same eye significantly increases its duration of visibifity if they are both in the same orientation. Thus, two processes are operating to generate the present pattern of results: interocular suppression and monocular, orientation-specific facilitation. Atkinson (1972) came to a similar conclusion from her work with paired afterimages, as did Wade (1973) from a study of binocular rivalry between paired afterimages. The interocular suppression effects do not appear to be orientation-specific, but Wade (1974) found that binocular rivalry between a vertical and a $45-\mathrm{deg}$ grating did yield significantly longer predominance durations for the vertical grating. That is, rivalry was shown to be influenced by orientation. It would seem that the dominance of a grating in binocular rivalry would require the mutual operation of two processes-local interocular suppression and intraocular contour facilitation. Since the evidence from the present studies suggests that interocular suppression is not orientation specific, it is likely that the contour facilitation effects operate more strongly for vertical than for oblique contours. Thus, the increased predominance of a vertical grating over a 45-deg grating in rivalry with it probably reflects the greater intraocular contour facilitation between the lines comprising the vertical as opposed to the 45 -deg gratings.

\section{REFERENCES}

Arkinson, J. Visibility of an afterimage in the presence of a second afterimage. Perception \& Psychophysics, 1972, 12. 257-262.

Atkinson, J. Properties of human visual orientation detectors: A new approach using patterned afterimages. Joumal of Experimental Psychology. 1973, 98, 55-63.

Bishop, P. O., \& HENRY, G. H. Spatial vision. Annual Review of Psychology. 1971, 22. 119-160.

BlaKe, R., \& Fox, R. The psychophysical enquiry into binocular summation. Perception \& Psychophysics. 1973, 14, 161-185.

Blakemore, C., \& ToBin, E. A. Lateral inhibition between orientation detectors in the cat's visual cortex. Experimental Brain Research. 1972, 15, 439-440.

ForDE, J. Binocular stimulation and the fragmentation of afterimages. 'Unpublished PhD thesis. University of Waterloo, 1971.

Julesz, B. Foundations of cyclopean perception. Chicago: University of Chicago Press, 1971.

Ogle, K. N. Binocular vision. New York: Hafner, 1964. 
RODGER, R. S. Intermediate statistics. Sydney: University Co-operative Bookshop, 1965.

WADE, N. J. Orientation effects on line afterimages. Perception \& Psychophysics, 1972, 12, 409-416.

WADE. N. J. Contour synchrony in binocular rivalry. Perception \& Psychophysics, 1973, 13, 423-425.

W ADE. N. J. The effect of orientation in binocular contour rivalry of real images and afterimages. Perception \& Psychophysics, 1974, 15, 227-232.
NOTE

1. The visibility periods were calculated for each subject in terms of duration/frequency, and then averaged. The averaged values for the visibility periods so calculated are given in Table 1 , and in the subsequent tables. These values do not, of course, correspond exactly to the ratios of the mean durations and frequencies given in the tables.

(Received for publication February 19, 1975; revision accepted September 18, 1975.) 